\title{
Family-centred interventions by primary healthcare services for Indigenous early childhood wellbeing in Australia, Canada, New Zealand and the United States: a systematic scoping review
}

\author{
Janya McCalman ${ }^{1 *}$, Marion Heyeres², Sandra Campbell ${ }^{2}$, Roxanne Bainbridge ${ }^{1}$, Catherine Chamberlain ${ }^{3}$,
} Natalie Strobel ${ }^{4}$ and Alan Ruben ${ }^{5}$

\begin{abstract}
Background: Primary healthcare services in Australia, Canada, New Zealand and the United States have embraced the concept of family-centred care as a promising approach to supporting and caring for the health of young Indigenous children and their families. This scoping review assesses the quality of the evidence base and identifies the published literature on family- centred interventions for Indigenous early childhood wellbeing.

Methods: Fourteen electronic databases, grey literature sources and the reference lists of Indigenous maternal and child health reviews were searched to identify relevant publications from 2000 to 2015 . Studies were included if the intervention was: 1) focussed on Indigenous children aged from conception to 5 years from the abovementioned countries; 2) led by a primary healthcare service; 3) described or evaluated; and 4) scored greater than 50\% against a validated scale for family-centredness. The study characteristics were extracted and quality rated. Reported aims, strategies, enablers and outcomes of family-centredcare were identified using grounded theory methods.

Results: Eighteen studies (reported in 25 publications) were included. Three were randomised controlled studies; most were qualitative and exploratory in design. More than half of the publications were published from 2012 to 2015. The overarching aim of interventions was to promote healthy families. Six key strategies were to: support family behaviours and self- care, increase maternal knowledge, strengthen links with the clinic, build the Indigenous workforce, promote cultural/ community connectedness and advocate for social determinants of health. Four enablers were: competent and compassionate program deliverers, flexibility of access, continuity and integration of healthcare, and culturally supportive care. Health outcomes were reported for Indigenous children (nutritional status; emotional/behavioural; and prevention of injury and illness); parents/caregivers (depression and substance abuse; and parenting knowledge, confidence and skills); health services (satisfaction; access, utilization and cost) and community/cultural revitalisation.
\end{abstract}

Discussion and conclusion: The evidence for family-centred interventions is in the early stages of development, but suggests promise for generating diverse healthcare outcomes for Indigenous children and their parents/caregivers, as well as satisfaction with and utilisation of healthcare, and community/cultural revitalisation. Further research pertaining to the role of fathers in family-centred care, and the effects and costs of interventions is needed.

Keywords: Family-centred, Patient-centred, Indigenous, Maternal and child health, Health outcomes

\footnotetext{
* Correspondence: j.mccalman@cqu.edu.au

'Central Queensland University, Cairns, Australia

Full list of author information is available at the end of the article
} 


\section{Background}

Primary healthcare services have embraced the concept of family-centred models of care as one approach to improve health and preventive services for Indigenous children [1-3]. Family-centred approaches differ from traditional maternal and child healthcare which focus on the management of individual women's pregnancies and infants' health and development at healthcare clinics. Instead, family-centred care approaches provide support and care for the whole family, their lives and wellbeing concerns, often at the family's home.

This scoping review was conducted to inform the development for a Cochrane review protocol [3] by systematically searching, selecting and synthesizing existing knowledge to map key concepts, types of evidence, and gaps in research about family-centred healthcare [4]. As suggested by Dijkers [5], assessments of the quality of the primary studies are included to provide confidence that the implications of the review for policy, practice or patients are based on high quality research. The research question was: What is thecurrent evidence base for the impact of family-centred interventions on Indigenous earlychildhood health? Both the Cochrane and this scoping review were contracted by a Queensland regional Indigenous community controlled health service, Apunipima Cape York Health Council, to inform the implementation of their family-centred Baby One Program (Bainbridge R, McCalman J, Campbell C, Redman-MacLaren M, Vine K, Canuto K, Sewter J, MacDonald M: Growing a relational and responsive family health promotion program: A grounded theory evaluation of the Baby One Program, inpreparation).

In mainstream populations, many health care providers now recognise family-centred care and the related concept of patient-centred care as integral to patient health, satisfaction, and health care quality, and consider them to be the standard of child health care [6]. For example, the US Healthy People 2020 plan for children recommends that children with special health care needs should receive care in a "family-centred, comprehensive, coordinated system" [7]. There is evidence from mainstream settings that family-centred interventions have resulted in decreased depression rates and burden in carers, improved quality of life for the entire family and satisfaction with care, as well as greater health service effectiveness and efficiency with reduced cost [8].

The need for improved child healthcare for Indigenous populations is evidenced by persistent disparities in child health equity in Australia, Canada, New Zealand and the United States. Mortality rates are higher in the four countries for all Indigenous infants except Native Hawaiians; there are generally fewer children born with normal birthweights (between 2500 and $4500 \mathrm{~g}$ ); and childhood obesity rates are considerably higher for Indigenous than the general populations in each of these countries [1]. These disparities reflect the shared legacy of the impacts of colonisation in these countries; whereby exclusionary social policies have to varying degrees disrupted family relations, continuity and functioning [9].

Many Indigenous families deal with ongoing stressors, which can manifest inpsychological distress, grief, smoking and alcohol and drug misuse, mental illnesses, and/or violence; and thus their ability to nurture children [9]. In turn, families can experience issues such as lack of food security, child neglect, and the removal of children [10]. However, Indigenous families also commonly experience strengths, such as strong bonding capital associated with their inclusion of members of their extended families, and the influence of traditional cultural norms on child rearing practices [9]. These strengths provide opportunity upon which engagement in health promoting family-centred approaches with services can be built to support improvements both to family lifestyle factors but also on the upstream social determinants of Indigenous childrens' health and wellbeing [9].

Primary health care services in Indigenous communities, which are increasingly managed and delivered by Indigenous community controlled health services, have taken opportunities to develop and implement family-centred interventions to improve Indigenous child health. By ensuring that care is planned and implemented around the whole family, family-centred interventions have the potential to recognise and support Indigenous family functioning, that is, their communication, maintenance of relationships in healthy ways, decision making and problem solving [11]. Health services can also advocate to address system barriers to improved family health, such as for education, training, employment, and to child protection agencies.

There are differing definitions for family-centred healthcare, and consequently various approaches. Nixon [12] defined the delivery of family-centred care by health services as "a way of caring for children and their families within health services which ensures that care is planned around the whole family, not just the individual child/person, and in which all the family members are recognised as care recipients". Griew, Tilton, and Stewart [13] proposed a broader two-part definition of Indigenous family-centred healthcare as: 1) movingbeyond providing care to the individual patient, to seeing them as being embedded in a family and providing services on that basis; and 2) taking a life course approach, which, without neglecting adult health, focused specific attention on establishing early life resilience and advantages through an emphasis on child development. This paper reviews the state and quality of the evidence for family-centred healthcare delivered through primaryhealthcare services for Indigenous children (from conception to 5 years). The review objectives were:

1) Outline the extent of the current evidence base for family-centred interventions by primary healthcare 
services for Indigenous Australian, Canadian, New Zealander or United States early childhood wellbeing;

2) Examine the conditions which enable primary healthcare services to implement family centred interventions, and the strategies they use to do so;

3) Describe the outcomes of family-centred interventions for Indigenous early childhood wellbeing.

\section{Methods}

\section{Inclusion/Exclusion criteria}

Studies were included in this scoping review only if they were published in English from 1 January 2000 to 31 December 2015 inclusive. The start date of the review was taken from 2000 when the US formally recognised patient-centred care as a healthcare standard [14]. Publications were also included only if the study met each of the following four criteria:

1. Participants were Indigenous Australian, Canadian, New Zealander or United States children aged from conception to five years who received family-centred care. A child was considered to be Indigenous if they were identified by the family as Indigenous (one parent may have been non-Indigenous); 'Indigenous' was defined using the United Nations definition of self- identification and acceptance by the community as a member [2].

2. Evaluated or described a family centred intervention or theorised a family centred healthcare model. We used Nixon's [12] definition of family-centred healthcare and included: a) environmental interventions that maximise parental involvement and enhance child health or wellbeing;

b) communication interventions that include parents/ caregivers in collaborative care pathways, and/or reorganisation of health care to provide continuity of carers; c) educational interventions for parents/ caregivers or staff; d) counselling interventions such as brief interventions, home visiting and other approaches; and/or e) family support interventions such as flexible charging schemes for poor families, referrals to other community services, parent-to-parent support [15]. We included pregnancy care models only if the intervention continued beyond the standard postpartum period of six weeks to at least three months.

3. Intervention scored greater than $26 / 52$ points (50\%) against a validated scale for family-centredness $[15,16]$. The scale incorporated 13 criteria, clustered under three concepts: 1) family as a constant (family as a constant in child's life, recognising family strengths, collaboration between parents/caregivers and professionals, needs-based family support, flexible provision of health care, sharing information with families); 2) culturally responsive (culturally competent health care, respecting family diversity, providing financial support); 3) supporting family individuality \& need for different types of family support (respecting family coping methods, providing emotional support, family-to-family support, attending to the developmental needs of children and families). Each criteria were scored from zero (no evidence that the author(s) addressed, endorsed, or advocated adoption of adherence to the elements of family centred care either implicitly or explicitly) to four (numerous instances of explicit evidence that the author(s) advancedadoption or support of the elements of family-centred care).

4. Intervention was led by a primary healthcare service, defined broadly as healthcare providers involved in providing primary healthcare for Indigenous children.

\section{Search strategy}

In consultation with an expert librarian (KK), a four-step search strategy was implemented. Step one comprised a search of 14 electronic databases: MEDLINE, PsycINFO, CINAHL, Informit, Indigenous Australia, Indigenous Studies Bibliography, AIATSIS, ATSIHealth, APAIS- ATSIS, FAMILY-ATSIS, Informit Indigenous Collection, Campbell Library, Cochrane Library, and Sociological Abstracts. MESH headings included family or parents or infant or newborn or legal guardians or pregnancy, AND child health services or Maternal Health Services or Maternal-Child Nursing or Family Health or Midwifery or Family Practice or Primary Health Care or General Practice or Delivery of Health Care or Patient-Centered Care or Health Promotion or Patient Care Planning AND Oceanic Ancestry Group OR American Native Continental Ancestry Group. Step two comprised searches of the grey literature through five clearinghouses or websites of relevant organisations in each of the four countries: Australian Indigenous Health InfoNet, Australian Institute of Family Studies, Indigenous Knowledge Network for Infant, Child and Family Health (Canada), Li Ka Shing database at St. Michael's Hospital (Canada), and New Zealand Social Policy Evaluation and Research Unit. Search terms were: family-centred care AND children OR infant OR maternity OR trimester. Step three comprised a search of the reference lists of Indigenous maternal and child health systematic reviews. In step four, the authors of this study also drew on their knowledge of family-centred interventions.

\section{Identification, screening and inclusion of publications}

The combined searches were imported into a bibliographic citation management software, EndNote X7 with duplicates removed. Titles and abstracts of the remaining publication titles and abstracts were screened by one author (MH). A second author (JM) retrieved and 
screened titles and abstracts of the remaining publications; those which did not meet inclusion criteria were excluded. The full texts of the remaining publications were retrieved and screened by blinded reviewers (RB, SC, CC, MH, JM, $\mathrm{AR}$ ) and independent reviewers from Apunipima Cape York Health Council and Centre for Research Excellence for Improving Health Services for Aboriginal and Torres Strait Islander Children (ISAC) (KE, RM, MRM, LS, NS, $\mathrm{KT}, \mathrm{MW}$ ). Inconsistencies in reviewer assessments were resolved by consensus.

\section{Data extraction \& analysis}

The publications were grouped together under the name for the study. Data were extracted from the full texts for publication authorship, publication year, study design, year/s of data collection and outcome assessment interval, study setting, population and sample size. The quality of included quantitative studies was assessed by blinded reviewers (SC and $\mathrm{CC}$ ) using the Effective Public Health Practice Project quality assessment tool [17]. Qualitative studies were assessed by blinded reviewers (MH and JM) using the Critical Appraisal Skills Programme quality assessment tool [18]. The costing study was assessed by a health economist (IK) and author (JM) using the Joanna Briggs Institute critical appraisal checklist for economic evaluations.

The publications were then imported into NVIVO software and coded (by MH). Grounded theory methods were used to map the strategies and outcomes of family-centred interventions, as well as the contexts and conditions under which they develop [19]. Grounded theory methods are well suited to conducting exploratory scoping reviews, especially in areas like family-centred interventions for Indigenous early childhood health, which is complex and has not been reviewed comprehensively before [19].

We started by coding the studies (seven publications) with the strongest study designs; then continued to code and compare the concepts in the remaining studies [19]. As we progressively coded and compared the papers, we found common or similar groups of concepts that were then recoded as higher order categories [19]. For example, across diverse studies, we identified strategies of providing subsidised fruit and vegetables; providing daily hot nutritious lunches, food coupons and hampers and nutritional supplements. We coded this concept as "augmenting diet". As more papers were coded, similar concepts were identified, such as providing oral health products; and providing safe sleeping baskets. Consequently, we regrouped and re-categorised the earlier code as "value-adding to health through products". Axial coding was then used to sort which of the categories represented the aim, contexts, conditions, strategies and outcomes of the family- centred interventions and to identify the interrelations between these [20]. Through axial coding, for example, "value-adding to health through products" became part of a core strategy titled "supporting family behaviours and self-care". These analytic coding steps did not occur in a lineal order as described here, but were performed interactively, revisiting and refining concepts and categories as new insights occurred [19].

\section{Results}

A Preferred Reporting Items for Systematic Reviews and Meta-Analyses (PRISMA) flowchart is presented at Fig. 1 [21]. The process of identification, screening and inclusion of publications resulted in 18 included studies (25 publications). One study of the US Family Spirit intervention was reported in five publications [22-26]; the Australian Baby Basket program in three publications [27-29], the Australian Triple P parenting study intwo publications [30, 31]; and the remaining studies had one publication each.

\section{Characteristics of studies}

Thirteen of the included 25 publications (52\%) were published in the last four years (2012-2015). Eleven of the 18 studies were Australian (61\%); three Canadian (17\%); one from New Zealand (6\%); and three from the USA (17\%). Only 13/18 studies stated their setting; these being spread fairly evenly across urban (5/13 or $38 \%)$, rural (4/13 or $31 \%)$ and remote areas (4/13 or $31 \%)$ (Table 1$)$.

Twelve/18 studies reported more than one study population. The majority of studies targeted expectant women or new mothers. In order of frequency, other client groups were: Indigenous children, parents/caregivers and other family members and other community members and stakeholders. Program deliverers, in order of frequency, were: Indigenous health paraprofessionals/ workers, senior/Elder women who provided education or support, other health practitioners, senior/Elder men, and partnerships withresearchers. This diversity was related to the inclusivity of many family-centred approaches and the varied modes of their delivery.

\section{Study design}

There were three/18 randomised controlled studies (17\%), one controlled before and after study (5\%) and one mixed method evaluation (5\%) to test the impact of familycentred interventions on the quality and effectiveness of care. However, the remaining 13/18 studies (72\%) were non-comparison studies, including three uncontrolled before and after studies, seven exploratory qualitative studies two program descriptions and a protocol for a longitudinal study (Fig. 2).

\section{Study quality}

Only one/18 studies was rated of strong quality [22-26] (Table 1). This study randomised 322 participants to the US Family Spirit intervention or optimised standard 


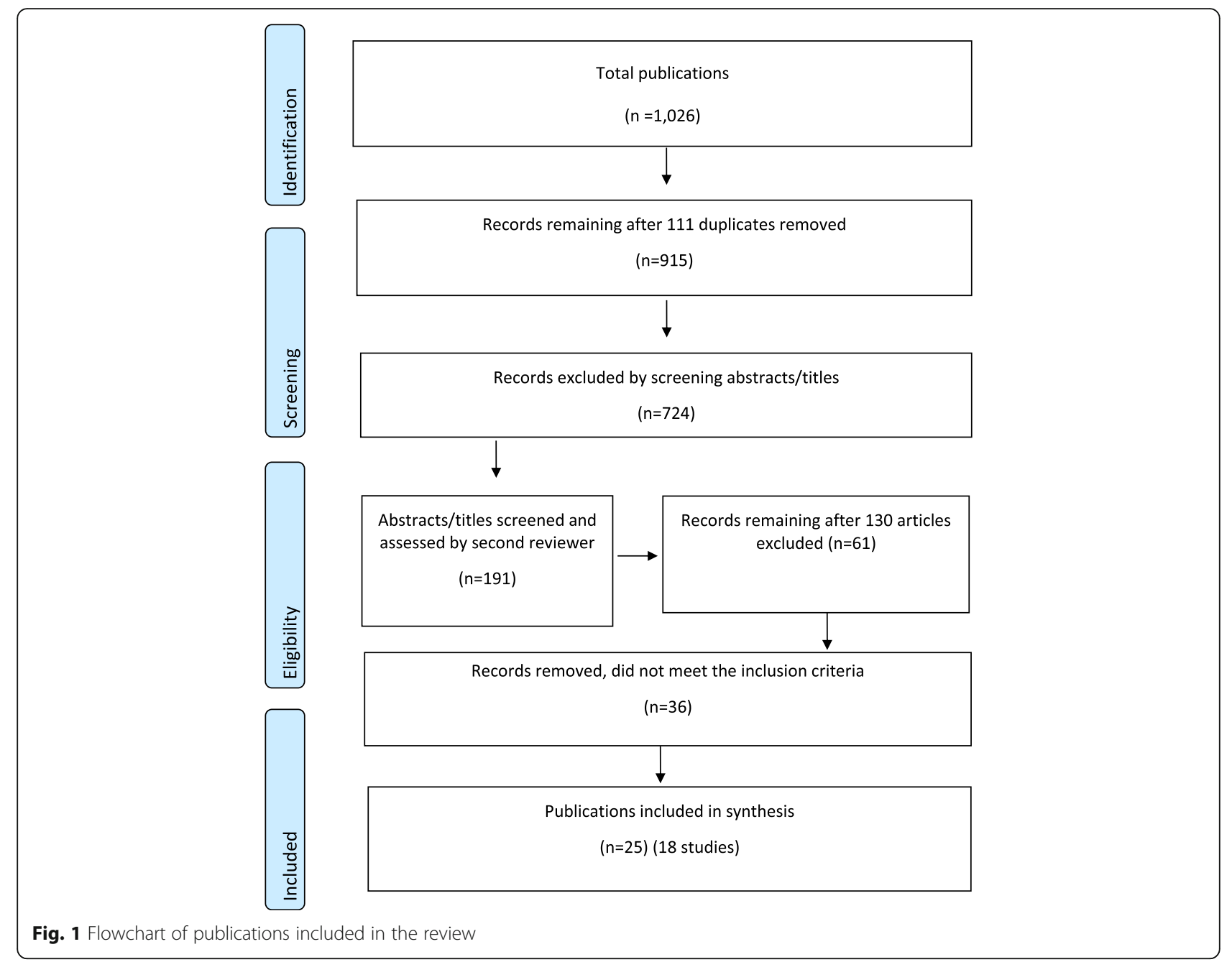

care, and evaluated outcomes at different time points using validated measurement tools. The other assessable studies were of moderate (7/18) moderate/weak (1/18), or weak (6/18) quality, with lack of consistently strong methodology across the majority of assessed criteria. The quality of two program descriptions and one study protocol were not assessed.

\section{Key elements of family-centred interventions}

The aims, strategies, conditions and outcomes of familycentred care reported in each study are summarised in Table 2, where $\checkmark$ denotes evidence that the author(s) advanced adoption or support of the element of familycentred care, $\sim$ denotes an implicit or inferred reference consistent with the intent of that element; and X denotes no evidence for that element of family-centred care.

\section{Aim of family-centred interventions}

The aim of study interventions was to promote healthy families; that is, to enable families to increase control over and to improve their health. In 14/18 studies (78\%), this aim was explicitly reported [22-42], and in the other four, it was inferred (Table 2). Examples of an explicit aim were to assess the effectiveness and cultural appropriateness of the Triple $\mathrm{P}$ parenting program [31]; and to evaluate the impact of a weekly subsidised box of fruit and vegetables [35]. Examples of an inferred aim were to determine family satisfaction with a family-centred service $[43,44]$ and to explore the views of service providers about how family-centred services work [45].

\section{Strategies of family-centred interventions}

Six key strategies were identified: supporting family behaviours and self-care, increasing maternal knowledge, linking with the clinic, building the Indigenous workforce, promoting cultural/ community connectedness and advocating for social determinants of health (Table 2). Intervention components varied, with many having multiple strategies.

\section{Supporting healthy family behaviours and self-care}

Fourteen studies (78\%) explicitly described or evaluated the provision of mentoring, counselling, advocacy and 


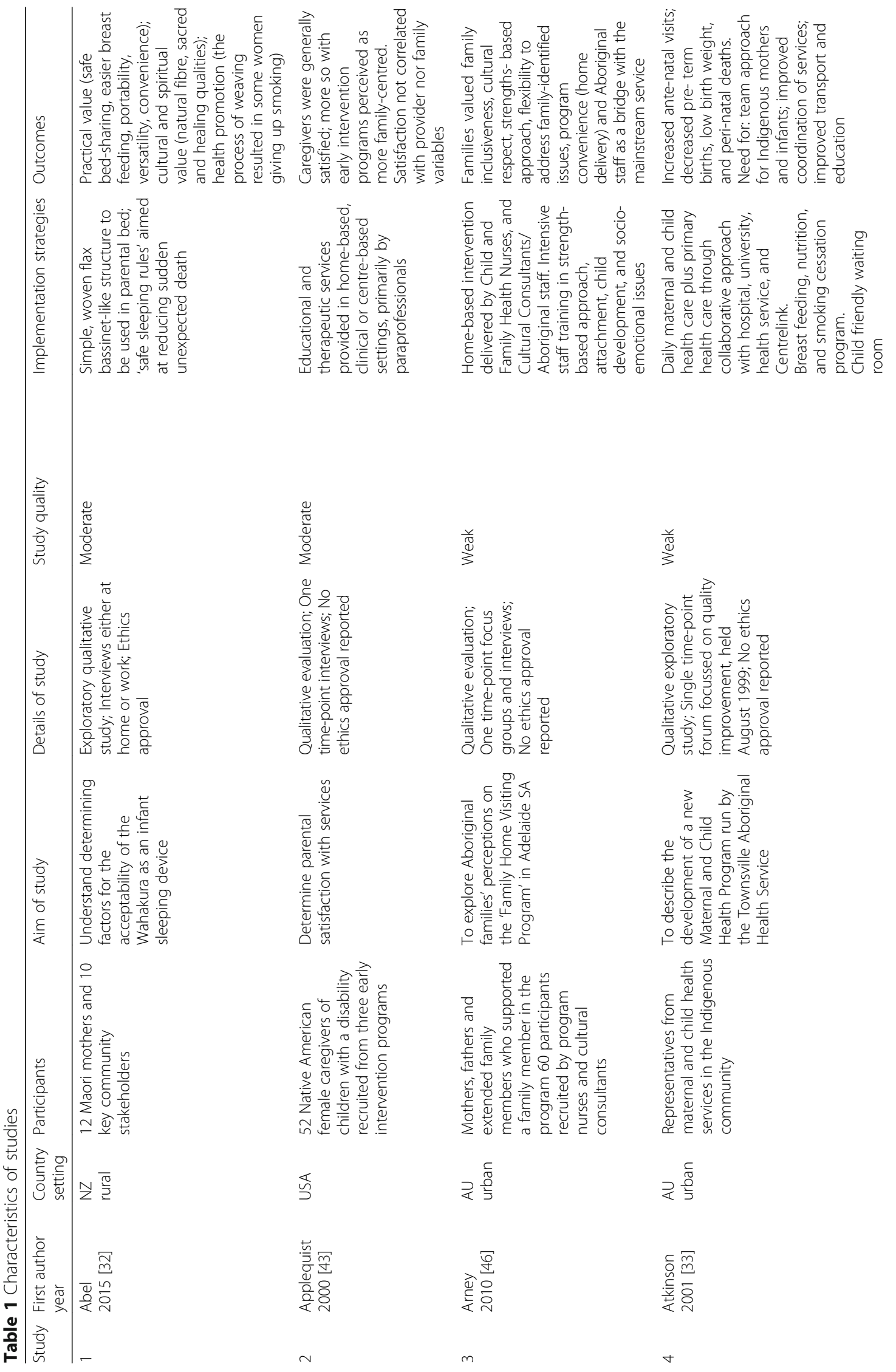




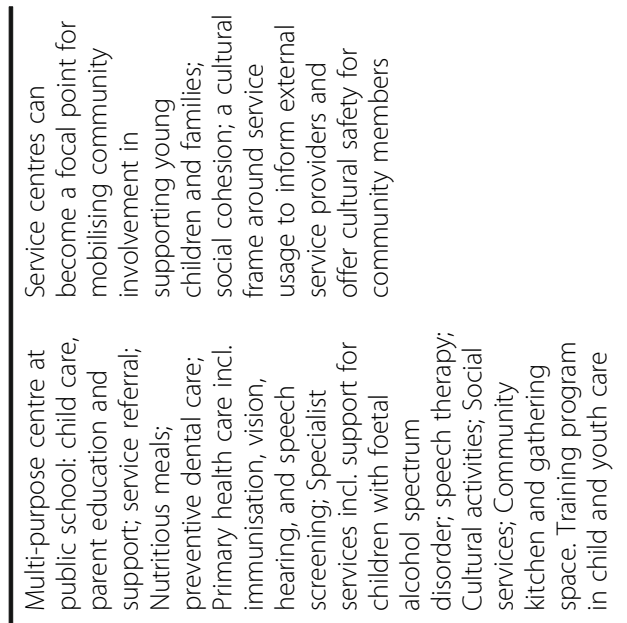

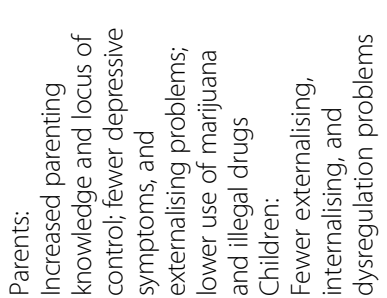

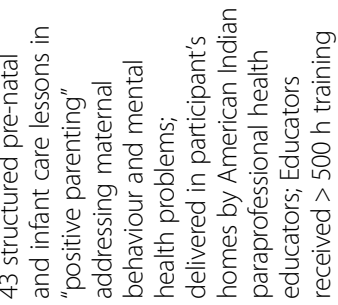

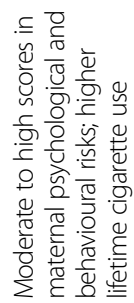

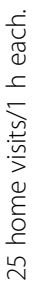

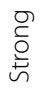

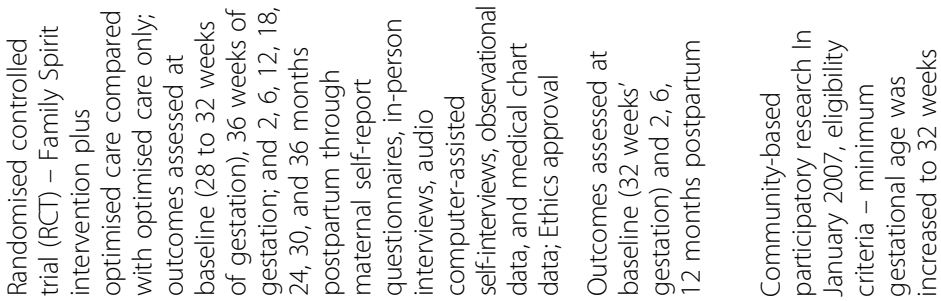

4

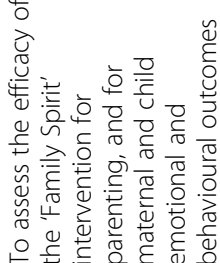

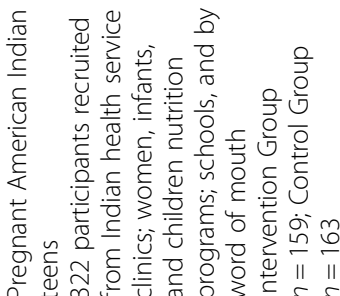

离

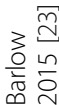

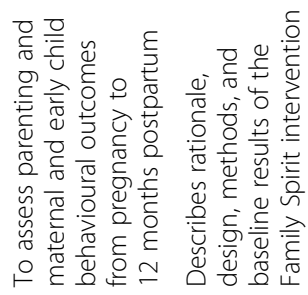

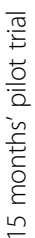




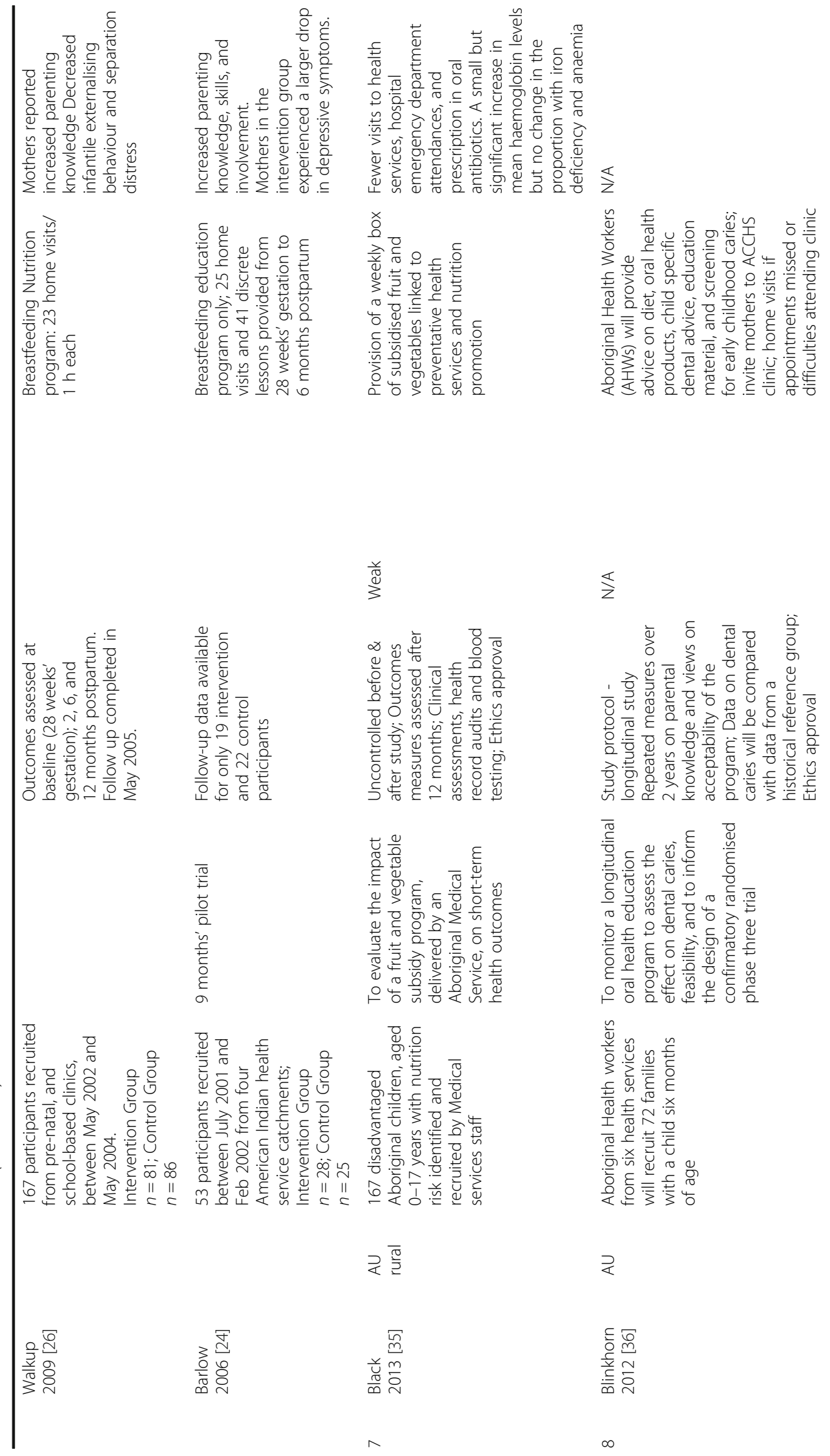




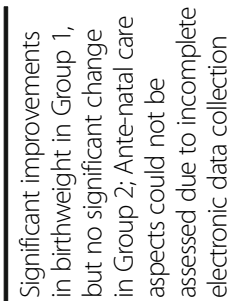

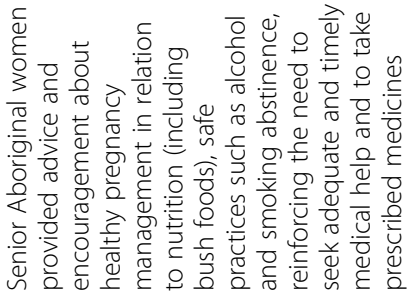
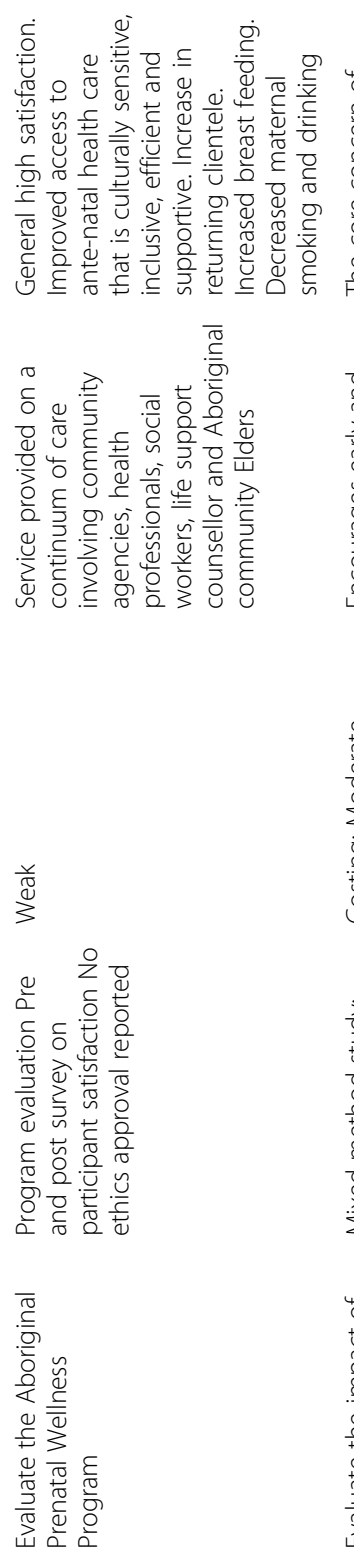

के

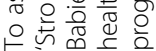

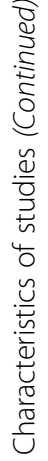

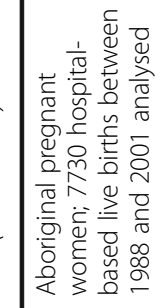

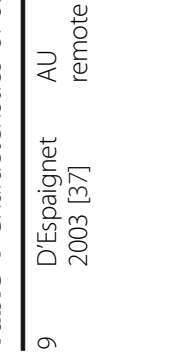

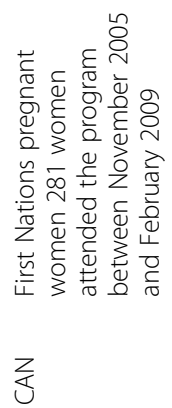

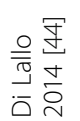

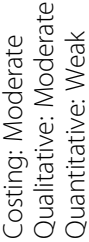

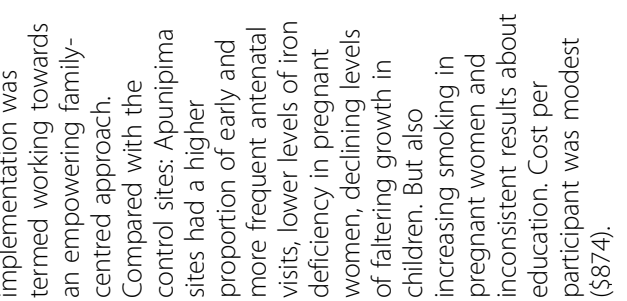

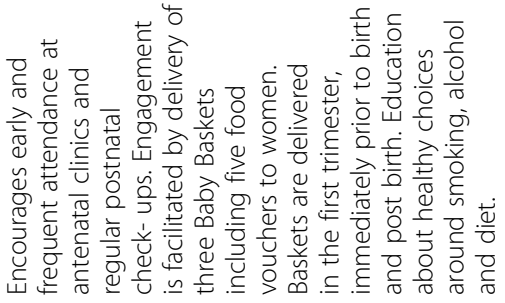

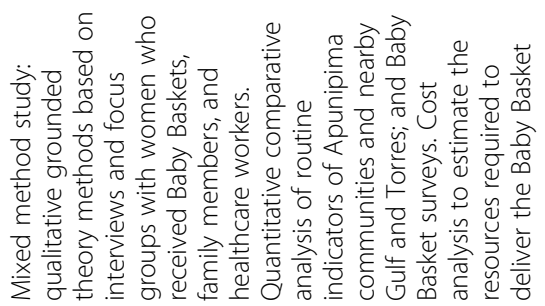

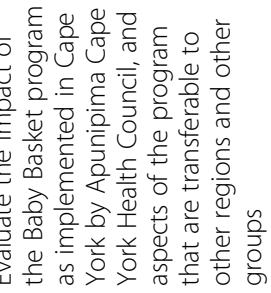

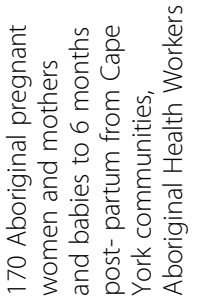

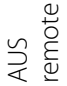

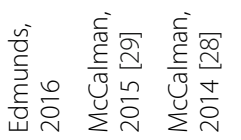




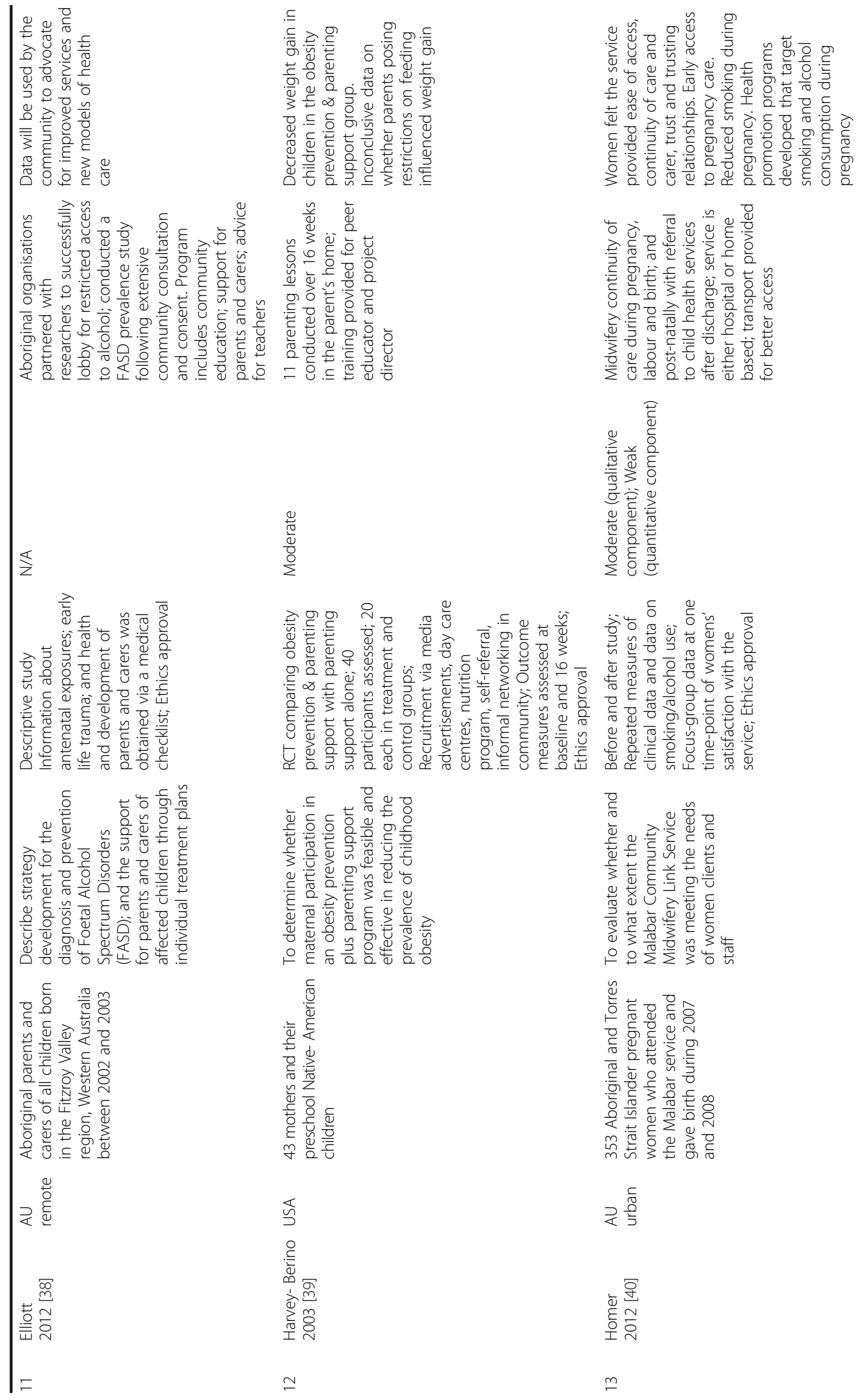




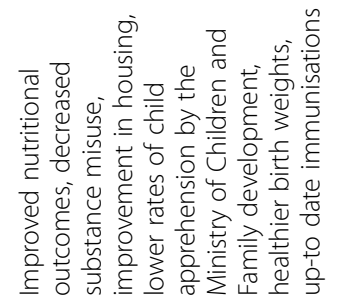

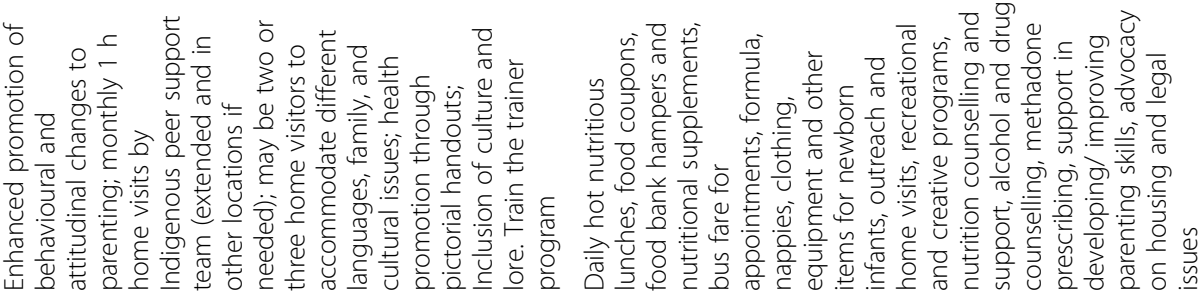

$\frac{\ll}{z}$

$\frac{\pi}{\frac{\pi}{4}}$

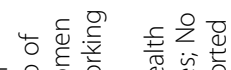

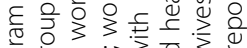

훙흐은

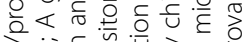

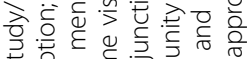

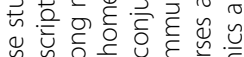

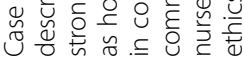

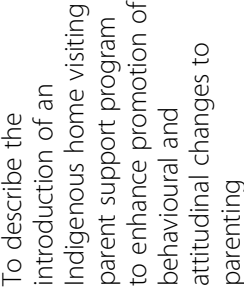

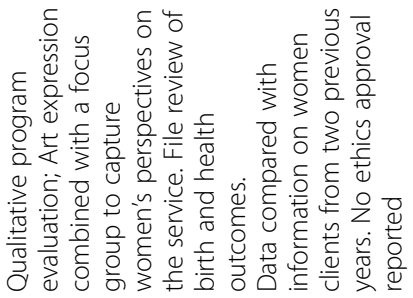

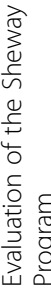

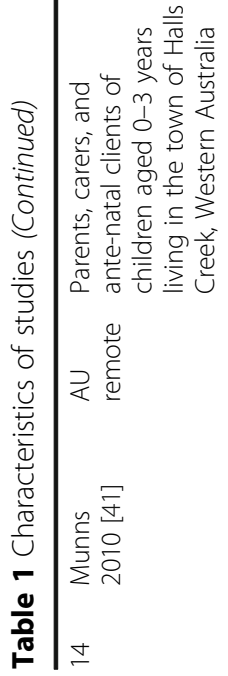

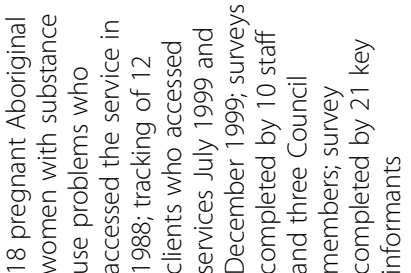

芯迹

일

$\stackrel{n}{\sim}$ 


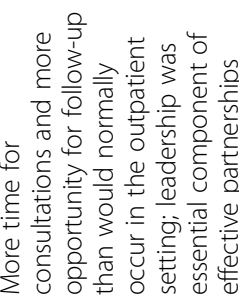

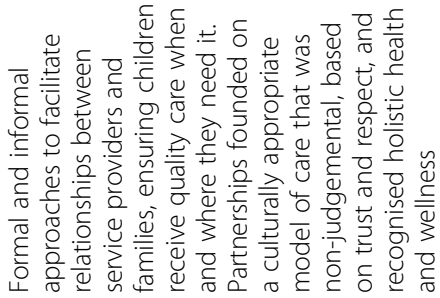

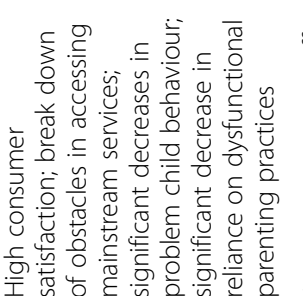

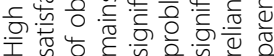
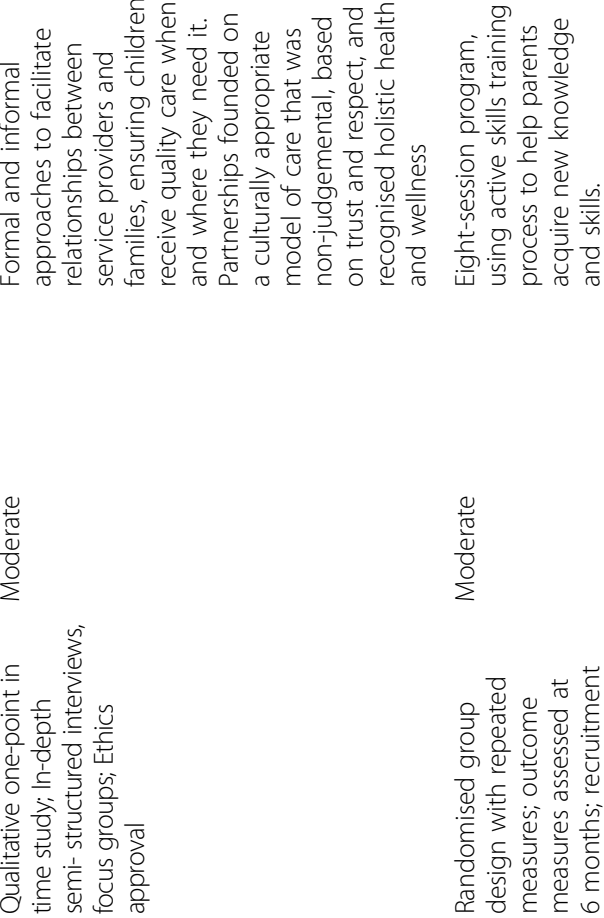

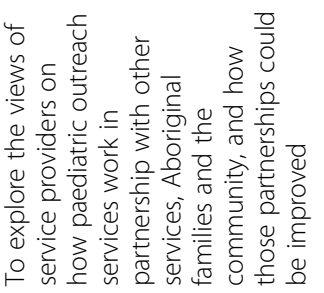

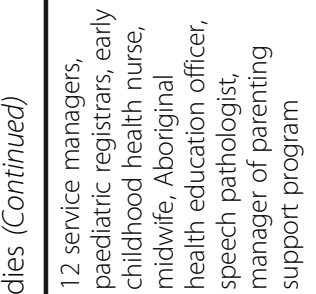

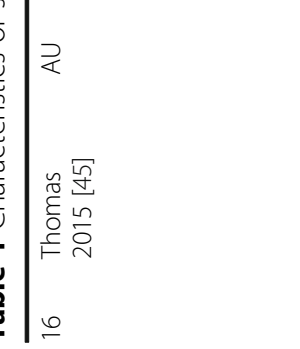

$\frac{0}{\frac{0}{0}}$
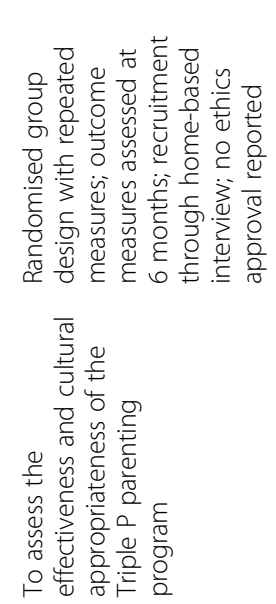

$\stackrel{\longleftarrow}{z}$
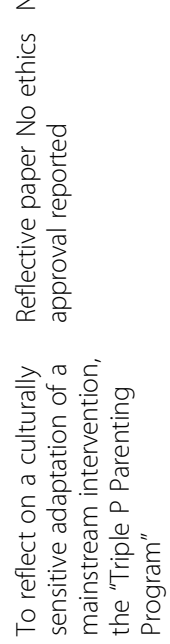

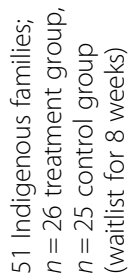

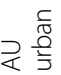

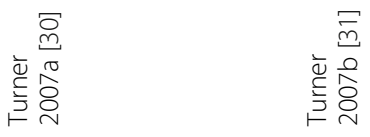

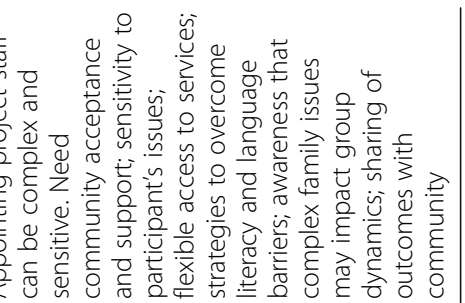

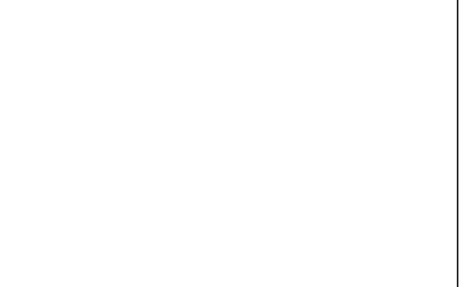




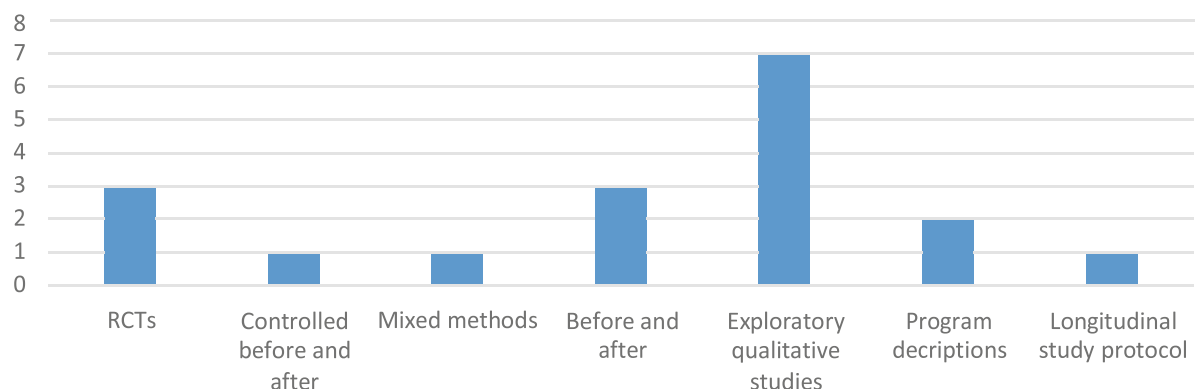

Fig. 2 Number of each type of study design

products to support healthy family behaviours and selfcare $[28-34,41-44,46]$. Mentoring by Indigenous Elders and/or health professionals was provided to encourage reduced or no alcohol use and smoking in pregnancy $[28,29,37,38,42,43]$; improve nutrition in pregnancy $[28,29,35,37,42,44]$; safe sleeping $[28,29,32]$; early childhood healthy eating and exercise routines to reduce obesity [28, 29, 39]; and care for and learning by disabled children [40]. As well, parents/caregivers were mentored to care for themselves [43] and reward themselves for meeting goals [36]. Counselling or brief interventions were provided to enhance nutrition and reduce alcohol and drug use $[28,29,42]$. Advocacy was also reported, for example to assist with housing, welfare and legal issues $[28,29,42]$ and for improved services and new models of healthcare [38].

Products, such as food and nutritional supplements, were provided to support women during pregnancy. For example, the Australian Baby Basket program provided antenatal, perinatal and postnatal baskets to Cape York women, which included a baby bed, educational books and clothing, nappies and other items for the baby and mother [29]. The Canadian Sheway program provided daily hot nutritious lunches, food coupons, food bank hampers and nutritional supplements for pregnant women struggling with substance abuse and addictions [42]. Products were also provided for new born infants, such as formula, nappies, clothing, and equipment such as sleeping baskets. Examples included the New Zealand Wahakura, a flax bassinet which was provided to promote safe sleeping for Maori infants [29], the Canadian Sheway program's provision of items for newborn infants [42], and an Australian Aboriginal Medical Service's provision of a weekly box of subsidised fruit and vegetables linked to preventative health services and nutrition promotion [35].

\section{Increasing maternal knowledge and skills}

All 18 studies (100\%) explicitly evaluated or described maternal health education and skills development. The foci of these strategies was to promote maternal skills generally, e.g. [22-26, 28-30], problem solving and coping skills [26, 31], goal setting [24, 46], breast feeding and nutrition skills $[28,29,39]$, dental health knowledge [36], safe sleeping [28, 29], smoking and alcohol reduction $[28,29]$ and the promotion of children's competence and development and management of misbehaviour [31]. Group or individual parent education was delivered in formal training or in home settings. To overcome literacy and language barriers, training was provided in intensive small group sessions or individually [26, 30, 31], and resources were made available in simple English, audio visually, and as table top flip charts [26, 28, 29, 31].

\section{Linking with the clinic}

Eight studies (44\%) explicitly reported linking families with clinical services [27, 28, 30, 31, 34, 37, 41, 42]. In some interventions, program educators encouraged family members to attend the health clinic for antenatal checks and birthing $[28,29,33,40,44]$, to seek timely medical help [31, 37], for immunization [28, 29, 34, 40], screening for vision, hearing and speech [34], and specialist paediatric services [34, 45].

\section{Building the Indigenous workforce}

Fourteen studies (78\%) reported employment, training and supervision of an Indigenous workforce as a strategy [22-26, 28, 29, 33-46]. For example, two newly graduated Aboriginal midwives were mentored through an urban Australian community midwifery service [40].

The Native American educators of the Family Spirit intervention were required to complete $500 \mathrm{~h}$ of training in home-visiting methods and curricular content, had to demonstrate competency in the form of written and oral examinations, and received daily on-site supervision and weekly cross-site conference calls [22-26]. Similarly, an Australian nurse home-visiting intervention provided extensive training for Aboriginal staff instrength-based approaches to attachment theory, child development and socio-emotional issues facing families $[28,29,46]$. 


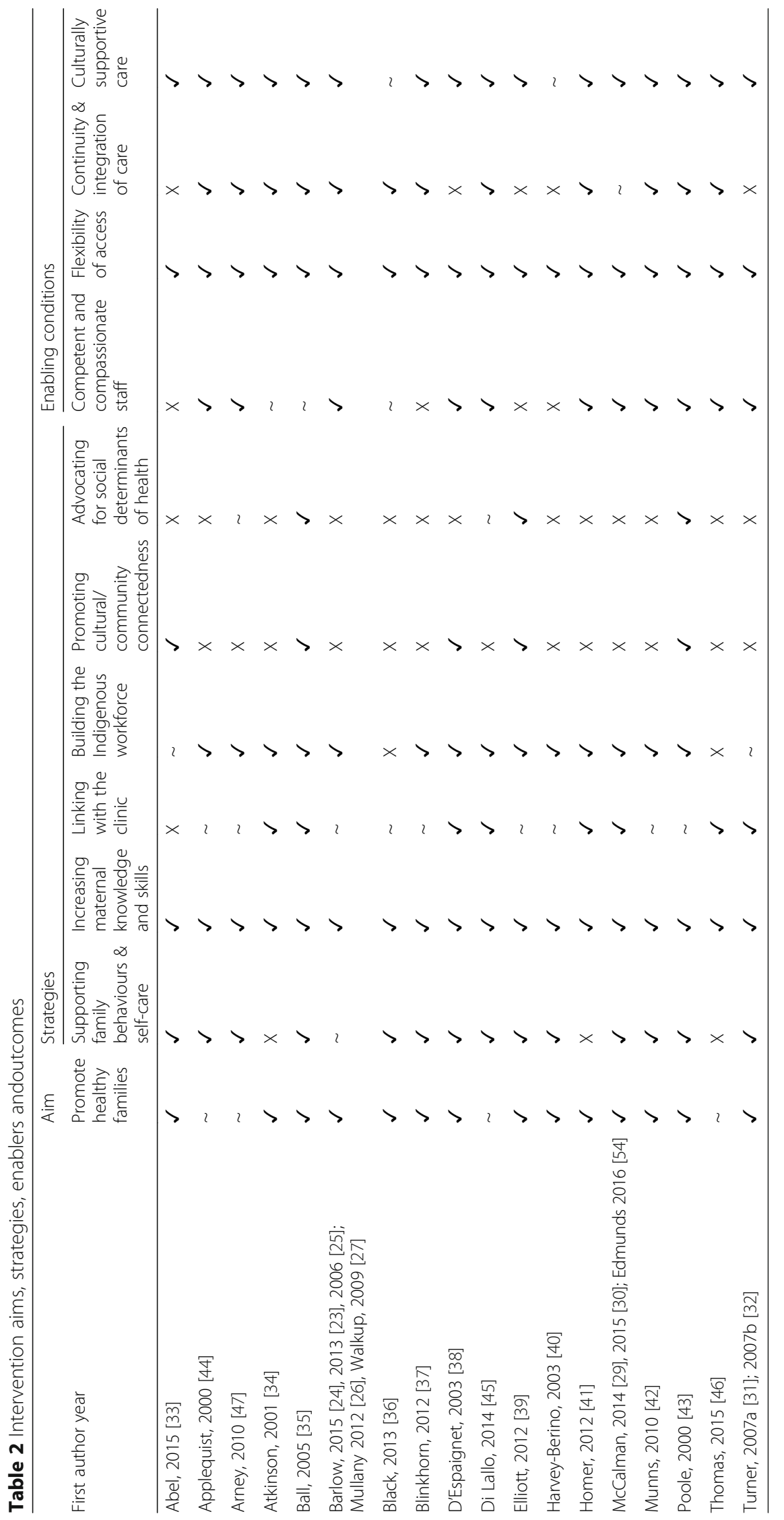




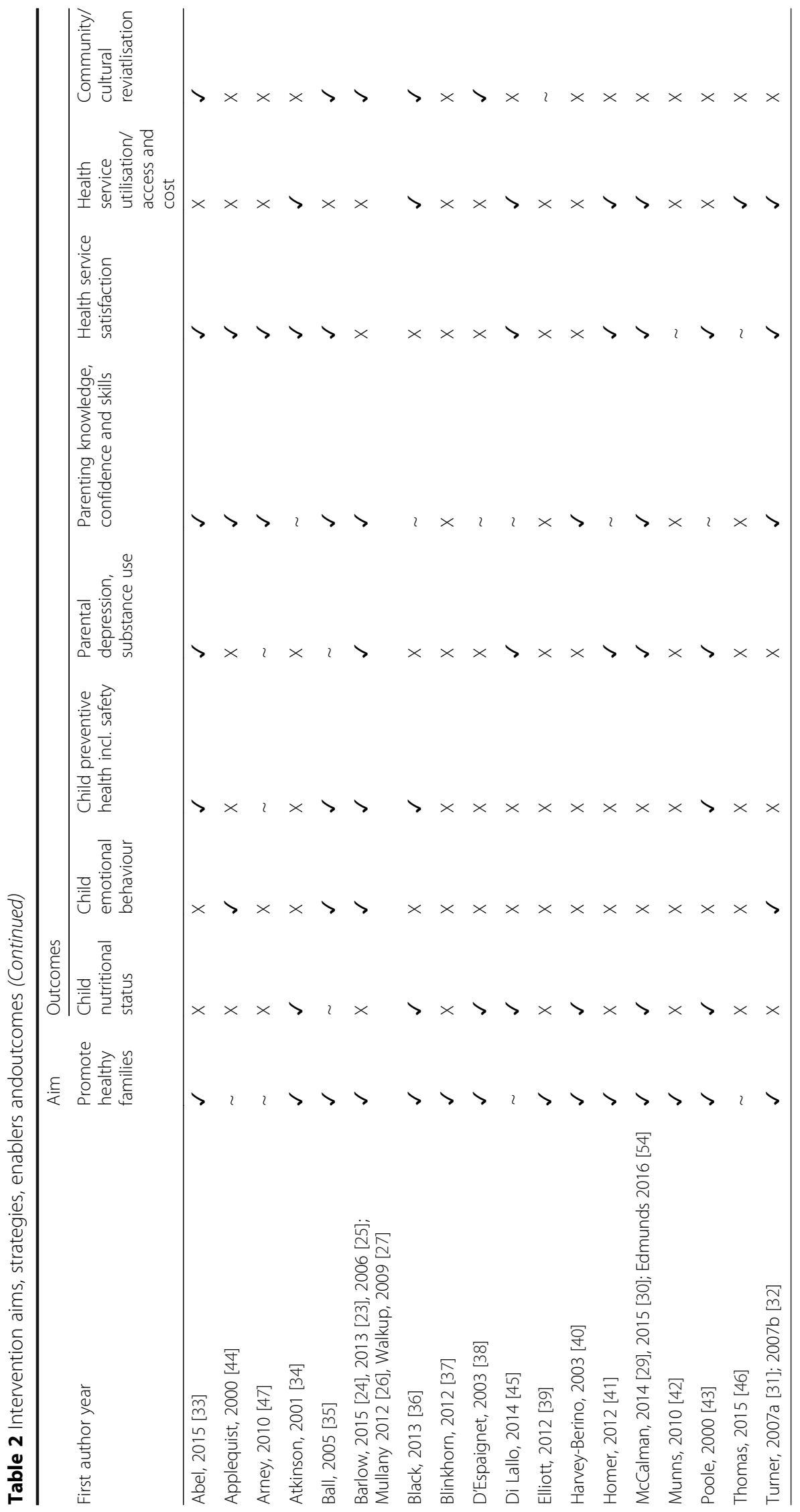




\section{Promoting cultural/Community connectedness}

Five studies (28\%) explicitly promoted cultural, spiritual or community connectedness as a strategy [32, 34, 37, 41, 42]. For example, new Maori parents were encouraged to use a safe sleeping device made from native flax, a material which had traditionally been used for weaving and was considered to have sacred and healing qualities [32]. Aboriginal Australian pregnant women were encouraged to make greater use of bush foods [37] and to become more engaged with local community events [41]. Canadian studies described early childhood care and development programs as a 'hub' for meeting a range of service and social support needs of community members [34] and encouraged pregnant women to identify a network of people whom they could call upon for support [42].

\section{Advocating for social determinants of health}

Three studies (17\%) described advocacy to improve aspects of the social and/or economic determinants of health [34, 38, 42]. Studies considered family-centred care to be a 'hook' for mobilising community involvement in supporting young children and families [34], advocated to restrict the sale of full-strength alcohol [38], and provided advocacy and support for child access and custody, other legal issues and housing [42].

\section{Enablers of family-centred interventions}

The four enablers of family-centred interventions were competent and compassionate program deliverers, flexibility of access, continuity and integration of care, and culturally supportive care (Table 2).

\section{Competent and compassionate program deliverers}

Eleven studies (61\%) cited the importance of having competent and compassionate staff as an enabler of familycentred care [22-26, 28-31, 37, 40-44, 46]. For example, Arney et al. [46] found that families' views about the program could not be separated from their appreciation of the qualities and abilities of the staff. Barlow et al. [22-24] required staff to have the ability to show compassion, be non-judgmental and have inter-personal skills.

Other publications emphasised the need for cultural sensitivity training to promote the interaction of practitioners with clients in ways that respected their cultural orientations and living situations [26, 30, 31]. Seven publications referred to the cultural competence of Indigenous program deliverers who could accommodate different languages, family and cultural issues [22-24, 28, 29, 37, 41, 42]. Homer et al. [40] however, found that it was the trusting relationship between provider and client that was important; this was not necessarily with an Aboriginal provider. Applequist \& Bailey [43] found that $96 \%$ clients indicated no preference regarding the ethnic background of their service provider.

\section{Flexibility of access}

Another hallmark of family-centred care interventions was the flexibility of access provided to health education and care. All 18 of the included studies (100\%) reported flexibility of access, including the provision of homebased care, e.g. [22-26, 28, 29, 31], choice of training location, e.g. [30, 31], or less commonly, the provision of transport or transport vouchers to and from services [40, 42]. Service providers considered it important to provide flexible access as an enabler of engagement, particularly to families without means of transport.

\section{Continuity and integration of healthcare}

Another enabler, reported in 12/18 studies (67\%), was the provision of healthcare continuity and integration by linking women across antenatal, birthing and postnatal services and providing integrated wrap-around care [22-26, 33-36, 40-46]. For example, Homer et al. [40] described a healthcare model whereby women were offered continuity of midwifery care during pregnancy, labour and birth; and referral to child health services postnatally after discharge.

Community agencies, health professionals, social workers, life support counsellors, and community Elders collaborated to provide integrated, wrap-around care for families [41, 44, 46]. Intercultural collaboration across Indigenous and mainstream health services was also considered important [44-46]. Leadership was considered an essential component of effective partnerships with other services, families and the community as it enhanced workplace ethos and created an environment where collaboration was supported [45].

\section{Culturally supportive care}

Culturally supportive care, based on secure, respectful and reciprocal relationships and partnerships with explicit respect for diversity, was highlighted in 16/18 studies (89\%) [22-26, 28-38, 40-46]. Being community driven, e.g. [38] or incorporating culture and lore, e.g. [41] was seen to enhance the effectiveness of programs and break down obstacles to accessing mainstream services, e.g. [31]. In some interventions, clients were provided a choice of the participants' native language or English for health education delivery $[22-26,41]$.

\section{Outcomes}

Intervention outcomes were reported in the $15 / 18$ evaluation studies (83\%) [22-26, 28, 29, 31-34, 37, 39, 40, 42-46] for Indigenous children, parents/caregivers, health services, and broader community/culture (Table 2). For Indigenous children, reported outcomes included improved nutritional status, emotional and behavioural and preventive health. For parents/caregivers of Indigenous children, studies reported reduced parental/caregiver 
depression and substance abuse, and improved parenting/ caregiving knowledge, confidence and skills. For health services, reported outcomes included client satisfaction and improved service utilisation and cost of delivery. Community/cultural revitalisation was also reported. Two studies that described programs $[38,41]$ and one study protocol [36] did not report outcomes.

\section{Child health outcomes}

\section{Children's nutritional status}

Seven/15 studies (47\%) reported improvements in children's nutritional status including changes in weight (overweight and underweight), growth and/or nutritional markers such as increased haemoglobin levels [28, 29, 33, 35, 37, 39, 42, 44]. Improved birth weights were reported following advice in relation to nutrition, alcohol and smoking during pregnancy, and utilisation of adequate and timely medical help [33, 37, 42]. Increased breast feeding was reported in a self-report survey following an Aboriginal Prenatal Wellness Program [44]. A reduced incidence of faltering growth was reported in an evaluation of the Australian Baby Basket program [28] and a small but significant increase in mean haemoglobin levels of children was found in a similar Australian study following the provision of a weekly box of subsidised fruit and vegetables linked to preventative health services and nutrition promotion [35]. Finally, decreased weight gain in children in the obesity prevention group of a US randomised controlled trial was found following an obesity prevention intervention with mothers of preschool Native-American children [39].

\section{Children's emotional behaviour}

Four/15 studies (27\%) reported improvements in children's emotional behavior [22-26, 31, 34, 43]. Improved coping strategies, self-expression and compliance were reported, as were lower rates of infant separation distress and child anxiety [22, 23, 26, 31, 42]. Fewer behavioural problems such as physical aggression, disobeying rules, fearfulness, separation distress, social withdrawal, or poorly modulated emotional responses in children were also found in the US 'Family Spirit' [22, 23, 26] and Australian Triple P [31] interventions.

\section{Preventing childhood injury and illness}

Five/15 studies (33\%) reported outcomes related to the prevention of childhood injury and illness [22-26, 32, 34, 35]. Improvements were found in attitudes toward, or actual home safety [22, 23, 32, 35]. For example, the US Family Spirit intervention resulted in an increased awareness of home safety issues in teen mothers [22, 23, 26]. The New Zealand Wahakura, a woven flax bassinet delivered with safe sleep messages, improved parental reassurance and confidence while providing the infant with a safe place to sleep in the parental bed [32]. The Canadian Sheway program resulted in housing improvement and lower rates of child apprehension by the Ministry of Children and Family development [42]. Studies also reported up to date immunisations [34, 42], screening for children's vision, hearing, and speech [34], and a significant decrease in prescribed oral antibiotics [35].

\section{Parent/carer outcomes \\ Parent/Carer's depression and substance misuse}

Six/15 studies (40\%) reported reductions in parental/ carer depression and/or substance misuse [22-24, 26, $28,29,32,40,42,44]$. For example, American Indian teen mothers had fewer externalising problems and depressive symptoms after participation in the Family Spirit intervention [22, 23]. Similarly, Poole [42] reported decreased substance misuse by pregnant women who participated in the Canadian Sheway program. Also reported were reductions in maternal smoking [32, 40, 42, 44] and use of marijuana and other illegal drugs [23, 42]. The Australian Baby Basket program was associated with a decrease in women who consumed alcohol during pregnancy over time. All women who consumed alcohol during pregnancy in 2013 were provided a brief intervention [28].

\section{Parenting/Caregiving knowledge, confidence and skills}

Eight/15 studies (53\%) reported improvements in parenting/caregiving knowledge, confidence and skills $[22-24,26,28,29,31,32,39,43,46]$. For example, improved parenting knowledge and locus of control were found in Native American teen mothers following the US Family Spirit intervention [22-24, 26]. Similarly, an Australian nurse- delivered home visiting program resulted in an improved sense of confidence in parenting [46]. Turner et al. [31] and Munns [41] found behavioural and attitudinal changes to parenting including a significant decrease in reliance on some dysfunctional parenting skills. The other five publications that explicitly aimed to enhance parental skills and practices were protocols or program descriptions and did not report outcomes.

\section{Health service outcomes \\ Satisfaction with healthcare}

Ten/15 studies (67\%) reported high satisfaction with family-centred health service provision $[28,29,31-35,40$, $42-44,46]$ with greater satisfaction reported for programs that were perceived to be more family-centred [43].

\section{Healthcare access, utilisation and cost}

Seven/15 studies (47\%) reported improved health access or utilisation as an outcome of family-centred care $[24,27-29,31,33,35,40,44,45]$. Culturally appropriate services were seen to promote more time for consultations and more opportunity for follow-up than 
would normally occur in an outpatient setting [28, 44, 45]. Also reported were earlier and increased utilisation of ante-natal care services $[28,33,40]$ and a breakdown of some of the obstacles Indigenous families faced in accessing mainstream services [31]. A reduction in visits to health services for illness, hospital emergency department attendances and oral antibiotic prescriptions was also found [35]. The Australian Baby Basket program evaluation reported that the cost per Baby Basket participant of about $\$ 874$ appeared to be a modest investment to provide babies with a better start in life [27].

\section{Community/cultural revitalization}

Finally, five/15 studies (33\%) reported community or cultural revitalisation as a result of implementing a family-centred intervention [22-24, 26, 32, 34, 35, 37]. The cultural and spiritual value of interventions was considered to be an outcome in its own right; for example, the Wahakura woven flax bassinet had cultural and spiritual value as well as promoting safe sleeping practices [32]. Centre-based interventions also became a focal hub for mobilising community involvement in supporting young children and families and encouraging social cohesion [34], as well as a basis to advocate for improved models of healthcare that offered cultural safety for community members [34, 38]. The employment of Indigenous para-professionals was also considered to have the potential to break multigenerational cycles of behavioural health disparities for Indigenous communities [22, 23, 26, 35, 37].

\section{Limitations}

Although a rigorous and thorough search strategy was used, it is possible that this scoping review did not locate all relevant studies. There was high level of agreement between blinded coders, and consensus on all included studies, but it is also possible that relevant intervention descriptions or evaluations may have been misclassified. Since evaluations with statistically significant findings are more likely to be published, it is possible that the published evaluations reviewed overestimate the true effectiveness of family-centred interventions in health care for Indigenous peoples [47].

\section{Discussion}

This review considered the current evidence base for the impact of family-centred interventions on Indigenous early childhood health. Like other reviews of Indigenous health $[48,49]$, we found little impact evaluation research that aimed to test the effectiveness of interventions, and only one study was rated of strong quality. The preponderance of the literature about familycentred interventions focussed on program descriptions or qualitative process evaluations, which explore the concepts and issues and described the interventions and formative or intermediate outcomes. It is likely that this is because the field is still in the relatively early stages of development, therefore there has not been enough elapsed time for follow-up studies and thus we do not know the full impact on Indigenous families of familycentred interventions.

The best evidence available suggest family-centred interventions can not only improve Indigenous children's health but also the health of their parents/caregivers. Studies suggest that outcomes include improved birth weights $[33,37,42]$ and reduced weight gain of obese children [39], reduced children's problem behaviours [22, 23, 26, 31], improved home safety, e.g. [23, 32, 42], and improved immunisation and screening rates [34, 35, 42].

Interventions also increased parenting knowledge [22, 24, 26, 31], involvement [24], locus of control [23], self-efficacy [22] and decreased reliance on some dysfunctional parenting practices [31]. Through improving parenting knowledge and skills, the interventions may have reduced the physical aggression of parents/caregivers $[22,23]$, depressive symptoms and past month use of marijuana and illegal drugs [23]. Health services experienced high rates of consumer satisfaction [31, 43], and improved access to mainstream services [31]. No adverse effects were reported. No study directly addressed the ultimate outcome of decreased morbidity as a result of the intervention.

A key gap in the evidence related to family engagement with and positioning in interventions. Familycentred care is based on the principle that parents bring expertise at both the individual care-giving level and the systems level [50]. However, few studies reported the extent to which families engaged in the family-centred interventions. Instead studies described the intervention components of a family-centred approach, focussed on their acceptability or feasibility, or users' satisfaction with services, or evaluated their health outcomes and/or costs. Thus MacKean's ([50] p. 81) observation of mainstream healthcare settings where; "family-centred care is beginning to sound like something that is being defined by experts and then carried out to families, which is ironic given that the concept of family-centred care emerged from a strong family advocacy movement" may also be apt in Indigenous settings. This finding may be related to use of a definition of family-centredcare developed for health service (rather than broader community) settings. However, the finding suggests that there is an important opportunity to develop a model of Indigenous family-centred care in the wider community context.

We found only three studies which considered the value of family-centred approaches in responding to the upstream social and economic determinants of Indigenous people's relatively poor health. The paucity of evidence in 
this area is of concern given the tendency identified by Popay et al. [51] for policies and programs to lifestyle drift; that is, to recognise the importance of the structural/ political determinants of health inequalities but to respond with action largely on behavioural lifestyle foci.

Another key gap identified in the reporting of intervention strategies pertained to the role of fathers in family-centred care. Ball [52] cited mother-centrism in parenting practices and child welfare services as barriers to positive involvement of Indigenous fathers with their children's health and wellbeing, yet none of the included studies explicitly considered the role of fathers. Further evaluation of the role of fathers in family-centred care interventions is needed through effective partnerships between primary healthcare services and research agencies to evaluate family-centred interventions as they roll out, thus minimising evaluation costs and optimising the use of locally available resources.

Only one study provided evidence of the costs of providing family-centred care to Indigenous families [27], and suggested that costs were offset by potential benefits. The paucity of economic evaluations was an identified gap in the scoping review. Another study of an intervention where senior Aboriginal women provided cultural support to pregnant women from remote Australian communities during labour, which was excluded from the review because it did not continue past one month post-partum, also found that the intervention was likely to be cost effective [53]. The finding suggests the potential for such interventions to be cost effective, but further such evaluations are needed.

A crucial issue in translating the results of this scoping review into policy or practice to inform interventions for improved Indigenous family health is that while the scoping study mapped the research and found 18 studies, these were generally of moderate to weak quality. This scoping review was conducted to produce a broad map of the evidence and to inform the scope and research objective of a Cochrane review protocol [3]. The Cochrane review will provide an independent and rigorous investigation, updated regularly to incorporate new research, of the best available evidence for the effects of family-centred interventions for children and their families. The Cochrane review will ensure that primary healthcare services can base their decisions about optimal interventions for the improvement of families' health on current and reliable evidence.

\section{Conclusion}

Family-centred interventions produced outcomes of improving Indigenous early childhood wellbeing, and the health of parents/ caregivers, as well as consumer satisfaction and improved access to mainstream services. The 18 studies evaluated or described the required conditions for implementing family-centred care to be the availability of competent and compassionate program deliverers, flexibility of access, continuity and integration of healthcare and culturally supportive care. Strategies were diverse and included supporting family behaviours and self-care, increasing maternal knowledge, strengthening links with the clinic, building the Indigenous workforce, promoting cultural or community connectedness and advocating for the social determinants of health. However, the evidence base for family-centred interventions by primary healthcare services is in an early stage of development, with few impact evaluation studies available. As well, there was little explanation in the available studies of how families engaged with and were positioned within family-centred interventions, whether or how interventions were able to impact the social determinants of families' health, the role of fathers in family-centred care and the costs of providing familycentred care. This scoping review informs the development of a Cochrane review protocol, which will provide regular updates of the available evidence as it develops.

\section{Abbreviations}

ISAC: Centre for Research Excellence for Improving Health Services for Aboriginal and Torres Strait Islander Children; PRISMA: Preferred Reporting Items for Systematic Reviews and Meta-Analyses

\section{Acknowledgements \\ Chief investigators and Apunipima staff members, Drs Mark Wenitong and Alan Ruben, approved the grant and the Apunipima Research Governance Committee approved the publication. Thanks to Katrina Keith who conducted the search of the electronic databases and websites, and the Apunipima, ISAC and associated colleagues who conducted the blinded screening of the publications. They were Karen Edmond, Rhonda Marriott, Michelle Redman-MacLaren, Linda Shields, Komla Tsey and Mark Wenitong. Thanks to Irina Kinchin who assessed the quality of the costing study. Thanks also to Karla Canuto, Research Co-ordinator at Apunipima Cape York Health Council, who provided critical feedback on the final draft of this paper.}

\section{Funding}

This research was funded by a grant from the National Health and Medical Research Council Centre for Research Excellence Improving Health Services for Aboriginal and Torres Strait Islander Children (ISAC) via Apunipima Cape York Health Council. The funding body played no role in the design of the study and collection, analysis, and interpretation of data or writing the manuscript.

\section{Availability of data and materials}

The data supporting our findings is provided in Table 1. A database of the search strategy records is available on request from the corresponding author.

\section{Authors' contributions}

JM led the scoping review, assessed the quality of qualitative studies and drafted the manuscript. MH developed Table 1, assessed the quality of qualitative studies, coded the data and contributed to drafting the manuscript. SC and CC assessed the quality of quantitative studies. RB, NS and AR made substantial contributions to conception and design of the review. All authors screened the publications, revised the manuscript critically for intellectual content, and read and approved the final manuscript.

\section{Authors' information}

SC, RB and CC are Aboriginal Australian researchers. JM, MH, NS and AR are non-Indigenous. 


\section{Competing interests}

The authors declare that they have no competing interests.

\section{Consent for publication}

Not applicable.

\section{Ethics approval and consent to participate}

Not applicable.

\section{Author details}

${ }^{1}$ Central Queensland University, Cairns, Australia. ${ }^{2} J a m e s$ Cook University, Cairns, Australia. ${ }^{3}$ University of Melbourne, Melbourne, Australia. ${ }^{4}$ University of Western Australia, Crawley, Australia. ${ }^{5}$ Apunipima Cape York Health Council, Bungalow, Australia.

\section{Received: 28 October 2016 Accepted: 7 February 2017}

\section{Published online: 21 February 2017}

\section{References}

1. Anderson I, Robson B, Connolly M, Al-Yaman F, Bjertness E, King A, et al. Indigenous and tribal peoples' health (The Lancet-Lowitja Institute Global Collaboration): a population study. Lancet. 2016;388:131-57.

2. United Nations. State of the World's Indigenous Peoples. New York: Indigenous Peoples' Access to Health Services; 2016.

3. McCalman J, Campbell SK, Chamberlain C, Strobel NA, Bainbridge RG, Wenitong $\mathrm{M}$ et al. Family- centred interventions for Indigenous early childhood well being by primary healthcare services (Protocol). Cochrane Database Syst Rev. 2016

4. Colquhoun HL, Levac D, O'Brien KK, Straus S, Tricco AC, Perrier L, et al. Scoping reviews: time for clarity in definition, methods, and reporting. J Clin Epidemiol. 2014;67(12):1291-4.

5. Dijkers M. What is a Scoping Review? KT Update. 2015:4:1.

6. Kuo DZ, Houtrow AJ, Arango P, Kuhlthau KA, Simmons JM, Neff JM. Family-centered care: current applications and future directions in pediatric health care. Matern Child Health J. 2012;16(2):297-305.

7. US Department of Human Services. Healthy People. US Department of Human Services. Washington, DC. 2016. https://www.healthypeople.gov/. 2016. Accessed 13 Feb 2017.

8. Bamm EL, Rosenbaum P. Family-centered theory: origins, development, barriers, and supports to implementation in rehabilitation medicine. Arch Phys Med Rehabil. 2008;89(8):1618-24.

9. Smylie J, Adomako P. Indigenous children's health report. Centre for Research on Inner City Health. 2009.

10. Libesman T. Child welfare approaches for Indigenous communities: International perspectives. Toronto: Keenan Research Centre, The Centre of Research on Inner City Health; 2004. p. 11-66. http://www.crich.ca. Accessed 12 Feb 2017.

11. Silburn S, Zubrick S, De Maio J, Shepherd C, Griffin J, Mitrou F, et al. The Western Australian Aboriginal Child Health Survey: strengthening the capacity of Aboriginal children, families and communities. Perth: Curtin University of technology and telethon institute for child health research; 2006

12. Nixon J. Family cohesion in families with an impaired child. Brisbane: University of Queensland; 1989.

13. Griew R, Tilton E, Stewart J. Family centred primary health care: review of evidence and models funded by the Office for Aboriginal and Torres Strait Islander Health Department of Health and Ageing. Canberra: Robert Griew Consulting with JTAI Pty Ltd.; 2007.

14. Institute of Medicine. Crossing the Quality Chasm: A new Health System for the 21st Century. Brisbane: The National Academies Press; 2001.

15. Shields L, Zhou H, Pratt J, Taylor M, Hunter J, Pascoe E. Family-centred care for hospitalised children aged $0-12$ years. Washington: The Cochrane Library; 2012. p. 1-59.

16. Trivette CM, Dunst CJ, Allen S, Wall L. Family-centeredness of the Children's Health Care journal. Child Health Care. 1993;22(4):241-56.

17. Effective Public Health Practice Project. Quality assessment tool for quantitative studies. 2004. Accessed 2 Sept 2016

18. Critical Appraisal Skills Programme. Critical Appraisal Skills Programme: making sense of evidence. Oxford: Better Value Healthcare Ltd; 2013. http://www.casp-uk.net/. Accessed 02 Sept 2016.
19. Wolfswinkel JF, Furtmueller E, Wilderom CP. Using grounded theory as a method for rigorously reviewing literature. Eur J Inf Syst. 2013:22(1):45-55.

20. Strauss A, Corbin J. Basics of qualitative research: techniques and procedures fordeveloping grounded theory. Thousand Oaks: Sage Publications, Inc; 1998.

21. Moher D, Liberati A, Tetzlaff J, Altman DG. Preferred reporting items for systematic reviews and meta-analyses: the PRISMA statement. Ann Intern Med. 2009;151(4):264-9.

22. Barlow A, Mullany B, Neault N, Compton S, Carter A, Hastings R, et al. Effect of a paraprofessional home-visiting intervention on American Indian teen mothers' and infants' behavioral risks: a randomized controlled trial. Am J Psychiatry. 2013;170:83-93.

23. Barlow A, Mullany B, Neault N, Goklish N, Billy T, Hastings R, et al. Paraprofessional-delivered home-visiting intervention for American Indian teen mothers and children: 3-year outcomes from a randomized controlled trial. Am J Psychiatry. 2015:172:154-62.

24. Barlow A, Varipatis-Baker E, Speakman K, Ginsburg G, Friberg I, Goklish N, et al. Home-visiting intervention to improve child care among American Indian adolescent mothers: a randomized trial. Arch Pediatr Adolesc Med. 2006:160(11):1101-7.

25. Mullany B, Barlow A, Neault N, Billy T, Jones T, Tortice I, et al. The Family Spirit Trial for American Indian teen mothers and their children: CBPR rationale, design, methods and baseline characteristics. Prev Sci. 2012:13(5):504-18. doi:10.1007/s11121-012-0277-2.

26. Walkup JT, Barlow A, Mullany BC, Pan W, Goklish N, Hasting R, et al. Randomized controlled trial of a paraprofessional-delivered in-home intervention for young reservation-based American Indian mothers. J Am Acad Child Adolesc Psychiatry. 2009;48(6):591-601.

27. Edmunds K, Searles A, Neville J, Ling R, McCalman J, Mein J. Apunipima Baby Basket Program: a retrospective cost study. BMC Pregnancy Childbirth. In press.

28. McCalman J, Edmunds K, Jongens C, Wargent R, Bainbridge R, Ling R, et al. Evaluating the Baby Basket program in north Queensland: as delivered by Apunipima Cape York Health Council, 2009 to 2013. 2014.

29. McCalman J, Searles A, Bainbridge R, Ham R, Mein J, Neville J, et al. Empowering families by engaging and relating Murri way: a grounded theory study of the implementation of the Cape York Baby Basket program. BMC Pregnancy Childbirth. 2015;15(1):1.

30. Turner K, Sanders M. Family intervention in Indigenous communities: emergent issues in conducting outcome research. Australas Psychiatry. 2007;15(6):S39-43. 1p.

31. Turner KM, Richards M, Sanders MR. Randomised clinical trial of a group parent education programme for Australian Indigenous families. J Paediatr Child Health. 2007:43(6):429-37.

32. Abel S, Stockdale-Frost A, Rolls R, Tipene-Leach D. The wahakura: a qualitative study of the flax bassinet as a sleep location for New Zealand Maori infants. N Z Med J. 2015;128(1413):12-9.

33. Atkinson $\mathrm{R}$, editor. Antenatal care and perinatal health-how to do it better in an urban Indigenous community. Canberra: Proceedings of the 6 th National Rural Health Conference; 2001

34. Ball J. Early childhood care and development programs as hook and hub for inter-sectoral service delivery in First Nations communities. J Aboriginal Health. 2005;1(2):36-50.

35. Black AP, Vally H, Morris PS, Daniel M, Esterman AJ, Smith FE, et al. Health outcomes ofa subsidised fruit and vegetable program for Aboriginal children in northern New South Wales. Med J Aust. 2013;199(1):46-50.

36. Blinkhorn F, Brown N, Freeman R, Humphris G, Martin A, Blinkhorn A. A phase II clinical trial of a dental health education program delivered by aboriginal health workers to prevent early childhood caries. BMC Public Health. 2012;12:681. http://dx.doi.org/10.1186/1471-2458-12-681.

37. D'Espaignet ET, Measey ML, Carnegie MA, Mackerras D. Monitoring the 'Strong Women, Strong Babies, Strong Culture Program': the first eight years. J Paediatr Child Health. 2003;39(9):668-72.

38. Elliott E, Latimer J, Fitzpatrick J, Oscar J, Carter M. There's hope in the valley. J Paediatr Child Health. 2012;48(3):190-2. http://dx.doi.org/10.1111/ j.1440-1754.2012.02422.x

39. Harvey-Berino J, Rourke J. Obesity Prevention in Preschool Native-American Children: A Pilot Study Using Home Visiting. Obes Res. 2003;11(5):606-11. http://dx.doi.org/10.1038/oby.2003.87

40. Homer CS, Foureur MJ, Allende T, Pekin F, Caplice S, Catling-Paull C. 'It's more than just havinga baby' women's experiences of a maternity service for Australian Aboriginal and Torres Strait Islander families. Midwifery. 2012;28(4):E449-55. http://dx.doi.org/10.1016/j.midw.2011.06.004. 
41. Munns A. Yanan Ngurra-ngu Walalja Halls Creek Community Families Programme. Neonatal. Paediatr Child Health Nurs. 2010;13(1):18-21. 4p.

42. Poole N. Evaluation report of the Sheway Project for high-risk pregnant and parenting women. Vancouver: British Columbia Centre of Excellence for Women's Health; 2000.

43. Applequist KL, Bailey Jr DB. Navajo caregivers' perceptions of early interventionservices. J Early Interv. 2000;23(1):47-61. http://dx.doi.org/ 10.1177/10538151000230010901.

44. Di Lallo S. Prenatal care through the eyes of Canadian Aboriginal women. Nurs Womens Health. 2014;18(1):38-46. doi:10.1111/1751-486X.12092. 9p.

45. Thomas S, Williams K, Ritchie J, Zwi K. Improving paediatric outreach services for urban Aboriginal children through partnerships: views of community-based service providers. Child Care Health Dev. 2015;41(6):836-42. doi:10.1111/ cch.12246. 7p.

46. Arney F, Bowering $K$, Chong A, Healy V , Volkmer B. Sustained nurse home visiting with families of Aboriginal children. Working with vulnerable families: A partnership approach. New York: Cambridge University Press; 2010. p. 109-34

47. Easterbrook PJ, Gopalan R, Berlin J, Matthews DR. Publication bias in clinical research. Lancet. 1991;337(8746):867-72

48. Paul $\mathrm{CL}$, Sanson-Fisher R, Stewart J, Anderson AE. Being sorry is not enough: the sorry state of the evidence base for improving the health of indigenous populations. Am J Prev Med. 2010;38(5):566-8.

49. Sanson-Fisher RW, Campbell EM, Perkins JJ, Blunden SV, Davis BB. Indigenous health research:a critical review of outputs over time. Med J Aust. 2006;184(10):502.

50. Mackean G, Thurston W, Scott C. Bridging the divide between families and health professionals' perspectives on family-centred care. Health Expect. 2005;8:74-85.

51. Popay J, Whitehead M, Hunter DJ. Injustice is killing people on a large scale — but whatis to be done about it? J Public Health. 2010;32(2):148-9.

52. Ball J. Fathering in the shadows: Indigenous fathers and Canada's colonial legacies. Ann Am Acad Pol Soc Sc. 2009:624(1):29-48.

53. Gao Y, Gold L, Josif C, Bar-Zeev S, Steenkamp M, Barclay L, et al. A cost-consequences analysis ofa Midwifery Group Practice for Aboriginal mothers and infants in the Top End of the Northern Territory, Australia. Midwifery. 2014:30(4):447-55.

54. Edmunds K, Searles A, Neville J, Ling R, McCalman J, Mein J. Apunipima Baby Basket Program: a retrospective cost study. BMC Pregnancy \& Childbirth. 2016;16:337. doi:10.1186/s12884-016-1133-3.

\section{Submit your next manuscript to BioMed Central and we will help you at every step:}

- We accept pre-submission inquiries

- Our selector tool helps you to find the most relevant journal

- We provide round the clock customer support

- Convenient online submission

- Thorough peer review

- Inclusion in PubMed and all major indexing services

- Maximum visibility for your research

Submit your manuscript at www.biomedcentral.com/submit 